\title{
Antikorruption im Krankenhaus - Wo liegen die Fallstricke?
}

\section{Einführung}

Strafverfahren gegen Ärzte und Klinikverantwortliche werden inzwischen nicht mehr ausschließlich wegen des Vorwurfs fahrlässiger Tötung oder Körperverletzung, des Abrechnungsbetruges und der Untreue geführt. Auf den Gesundheitssektor spezialisierte Staatsanwälte und Polizeibeamte ermitteln seit Einführung der durch das Gesetz zur Bekämpfung von Korruption im Gesundheitswesen vom 30. Mai 2016 neu eingeführten Straftatbestände der $\S \S 299 a$, b StGB (Bestechung und Bestechlichkeit im Gesundheitswesen) nun auch wegen des Verdachts auf Korruption im medizinischen Bereich. Hinsichtlich der Tatbestandsvoraussetzungen der $\S \S 299 a$, b StGB verweisen wir auch auf die Stellungnahme der Deutschen Röntgengesellschaft e. V. (DRG) zu dem Entwurf eines Gesetzes zur Bekämpfung von Korruption im Gesundheitswesen (Fortschr Röntgenstr 2015; 187, DRG-Mitteilungen, S. 500 ff.).

Angestellte Ärzte sowie nichtärztliche Mitarbeiter in Krankenhäusern konnten sich wegen Bestechlichkeit und Bestechung bereits vor der Einführung der neuen Straftatbestände nach § 299 StGB (Bestechung und Bestechlichkeit im geschäftlichen Verkehr) strafbar machen. Die Strafbarkeit nach § 299a und b StGB tritt für angestellte Krankenhausärzte nunmehr neben die Strafbarkeit nach §299 StGB. Nach dem Willen des Gesetzgebers ist eine tateinheitliche Verwirklichung beider Straftatbestände möglich (BT-Drucks. 18/6446, 16). Die neuen Straftatbestände führen dazu, dass in der Vergangenheit durchaus übliche und über viele Jahre gelebte Kooperationen nunmehr mit Strafe bedroht sind und grundlegend auf den Prüfstand gestellt werden müssen. Dieser Beitrag soll die häufigsten Fallstricke aufdecken, denen Ärzte und Entscheider in Krankenhäusern seit der Reformierung des Antikorruptionsrechts ausgesetzt sind.

Kooperationen sind vom Gesetzgeber ausdrücklich erwünscht, wie es die Gesetzesbegründung zur Einführung der $\S \S 299 a, b$ StGB betont. Dies belegen beispielweise
Regelungen wie die des §115a Abs. 1 S. 2 SGB V, wonach das Krankenhaus die vorund nachstationäre Behandlung durch hierzu ausdrücklich beauftragte niedergelassene Vertragsärzte in den Räumen des Krankenhauses oder der Arztpraxis erbringen kann. Dennoch schwebt über zahlreichen Kooperationen nunmehr das Damoklesschwert der §§ 299a, b StGB.

\section{Zielvereinbarungen in Chefarztverträgen}

Zielvereinbarungen in Chefarztverträgen sind seit langem etabliert und nach den Empfehlungen der Deutschen Krankenhausgesellschaft gemäß §136a SGB V zu leistungsbezogenen Zielvereinbarungen grundsätzlich legitim und sachgerecht, sofern sie sich an den Vorgaben des $§ 23$ der Muster-Berufsordnung für Ärzte (bzw. den von den Landesärztekammern in den jeweiligen Bundesländern erlassenen Berufsordnungen) orientieren. Der Qualitätsbericht des Krankenhauses nach §136b Abs. 1 S. 1 Nr. 3 SGB V muss Angaben zu Zielvereinbarungen mit leitenden Ärzten beinhalten. Gerade Leistungen, die im Mindestmengenkatalog des $\S 136 \mathrm{~b}$ Abs. 1 S. 1 Nr. 2 SGB V enthalten sind, werden von Krankenhäusern häufig in Zielvereinbarungen aufgenommen und das Erreichen einer Mindestzahl wirtschaftlich belohnt. Damit die Unabhängigkeit der medizinischen Entscheidungen gewahrt bleibt, dürfen finanzielle Anreize nach den Empfehlungen der Deutschen Krankenhausgesellschaft jedoch nicht für einzelne Operationen oder Leistungen vereinbart werden. Neben berufsrechtlichen Folgen können Zielvereinbarungen, die einen finanziellen Anreiz setzen eine korruptionsrechtliche Relevanz erhalten, wenn - wie häufig in ländlichen Gebieten vorkommend - ein Chefarzt nicht in Vollzeit für das Krankenhaus tätig ist, sondern seiner Chefarzttätigkeit für das Krankenhaus lediglich im Rahmen des § 20 Ärzte-ZV neben seiner Tätigkeit als niedergelassener Arzt nachgeht. In dieser Konstellation ist es denkbar, dass der Radiologe in seiner Aufgabe als niedergelassener Arzt Patienten in das Krankenhaus zur dortigen

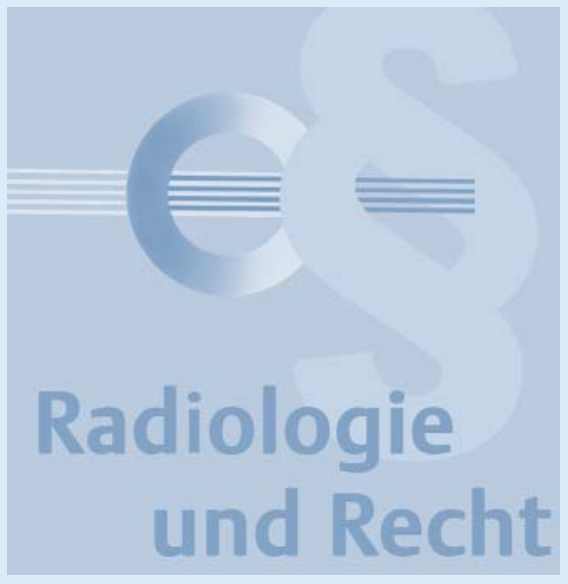

Behandlung durch sich selbst in seiner Funktion als Chefarzt zuweist. Durch die Verknüpfung mit einem für das Erreichen einer Mindestfallzahl seitens des Krankenhauses ausgezahlten Bonus‘, könnte der Arzt den Tatbestand der Bestechlichkeit erfüllen. Ob ein Gericht den gewährten Vorteil jedoch als rechtswidrig erachtet oder ob es die zugrundeliegende Zielvereinbarung als vertraglichen Anspruch auf die Bonuszahlung ausreichen lässt, bedarf einer Einzelfallprüfung, die auch die konkrete Höhe des in der Zielvereinbarung festgelegten Bonus‘ berücksichtigt.

\section{Honorararztverträge}

Probleme ergeben sich bei Kooperationen häufig im Zusammenhang mit pauschalen Vergütungssätzen und Exklusivitätsklauseln in Honorararztverträgen.

So kann beispielweise eine Vorteilsgewährung im Sinne des §299a, b StGB in Form der pauschalen Vergütung eines Honorararztes liegen. Die Höhe der Vergütung eines Honorararztes ist grundsätzlich anhand des persönlich erbrachten Leistungsanteils zu ermitteln. Zu fragen ist demnach, in welchem Verhältnis der Wert der honorarärztlichen Leistung zum Wert der Gesamtleistung steht. Die Kalkulation der DRGs ist dem Institut für das Entgeltsystem im Krankenhaus (InEK) übertragen. Trotz der Möglichkeit, eine von der Gebührenordnung abweichende Gebührenhöhe vertraglich festzulegen, kann das vereinbarte Honorar nicht beliebig hoch sein. So setzt insbesondere das ärztliche Berufsrecht der Höhe 
der zu vereinbarenden Gebühren Grenzen. Gemäß § 12 der Muster-Berufsordnung muss die Honorarforderung des Arztes angemessen sein. Der Arzt hat hierbei die besonderen Umstände des einzelnen Falles, insbesondere die Schwierigkeit der Leistung und den Zeitaufwand nach billigem Ermessen zu berücksichtigen. Kooperationsverträge, die eine pauschale Vergütung des Honorararztes vorsehen, etwa $20 \%$ der vom Krankenhaus abgerechneten DRGs, werden in vielen Fällen dazu führen, dass die Vergütungshöhe nicht leistungsangemessen ist. Je nach Behandlung kann der ärztliche Leistungsanteil höher oder aber auch deutlich geringer sein als der technische Leistungsanteil. Erbringt ein Honorararzt eine Vielzahl verschiedener Behandlungen mit deutlich abweichenden InEK-Kalkulationen hinsichtlich des ärztlichen und technischen Leistungsanteils, wird die pauschale Vergütung nicht für sämtliche erbrachten Leistungen angemessen sein. Ist die pauschale Vergütung im Einzelfall zu hoch angesetzt, kann hierin neben einem berufsrechtlichen Verstoß auch eine unzulässige Vorteilsgewährung liegen.

Im Rahmen eines von der LÄK Thüringen, der KV Thüringen und der Landeskrankenhausgesellschaft Thüringen durchgeführten „Runden Tisches zum Antikorruptionsgesetz“ (vgl. Ärzteblatt Thüringen 5/2017, S. 292) haben die teilnehmenden Vertreter der Staatsanwaltschaft erklärt, dass sie Kooperationsvereinbarungen zwischen niedergelassenen Ärzten und Krankenhäusern in erster Linie daraufhin überprüfen würden, ob ein Vorteil gewährt werde und eine Unrechtsvereinbarung vorliege. Die Angemessenheit der Vergütung solle dabei zunächst nicht im Vordergrund stehen. Sie hoben jedoch hervor, dass die im Rahmen eines Clearingverfahrens abgegebene Stellungnahme zur Kenntnis genommen werde, damit aber nicht der Strafbarkeitsvorwurf entfallen müsse. Die Staatsanwälte wiesen daraufhin, dass die Verträge transparent und somit für jeden nachvollziehbar gestaltet werden sollten. Ob sich auch weitere Staatsanwaltschaften im Bundesgebiet dieser Auffassung anschließen, bleibt abzuwarten.

Auch sog. Konkurrenzschutzklauseln kommen in vielen Kooperationsverträgen vor und bergen ein strafrechtliches Risiko. Für
§ 299a Nr. 1 StGB reicht in Nr. 3 die Zuführung von Patienten oder Untersuchungsmaterial aus. Eine Konkurrenzschutzklausel, die den Honorararzt verpflichtet, stationäre Leistungen ausschließlich am kooperierenden Krankenhaus durchzuführen und darüber hinaus selbst bei Patienten, die nicht durch den Honorararzt operiert werden, aber stationär behandelt werden müssen, darauf hinwirken, dass sich diese im kooperierenden Krankenhaus operieren lassen, ist unzulässig. Auch ein vermeintlich korrigierender Zusatz wie „unter Beachtung der freien Arztwahl“ ändert daran nichts. Ein solcher Zusatz steht im klaren Gegensatz zu dieser Forderung in der Konkurrenzschutzklausel. Die Zuführung von Patienten wird mit einer solchen Konkurrenzschutzklausel sogar schriftlich belegt.

\section{Belegarztverträge/ ambulantes Operieren}

Auch Belegarztverträge enthalten erfahrungsgemäß häufig Regelungen, die korruptionsrechtlich bedenklich sind. Beispielweise berechnen einige Krankenhäuser gegenüber einzelnen Belegärzten für bestimmte Operationen kein Nutzungsentgelt für die Überlassung von Räumlichkeiten, Personal und Instrumentarien. Dies erweckt den äußeren Anschein, dass die konkreten Belegärzte ihre Belegpatienten möglicherweise allein auf Grund eines finanziellen Vorteils in dem kooperierenden Krankenhaus behandeln. Die belegärztliche Kooperation stellt jedoch nur dann eine grundsätzlich zulässige Verdienstmöglichkeit dar, wenn ein angemessenes Entgelt für die durch das Krankenhaus zur Verfügung gestellten Nutzungsmöglichkeiten geleistet wird. Die Zahlung eines Nutzungsentgelts durch die betroffenen Belegärzte an das Krankenhaus ist zwingend geboten. Andernfalls wäre bei Erfüllung der weiteren Voraussetzungen der Tatbestand des § 299a bzw. b StGB verwirklicht, denn das willkürliche Erlassen eines Nutzungsentgelts stellt eine Vorteilsgewährung dar und kann auf eine Unrechtsvereinbarung zwischen dem Krankenhaus und dem kooperierenden Belegarzt hindeuten.

\section{Sponsoring von internen Fortbildungen}

Chefärzten in Krankenhäusern werden nicht selten von Medizinprodukte- und Pharmaherstellern Sponsorings und Referentenverträge angeboten. Dies kann beispielsweise ein Sponsoring einer krankenhausinternen Fortbildung in den Räumlichkeiten des Krankenhauses durch einen Kontrastmittelhersteller sein, bei der ein externer Referent zu Themen wie dem Notfall-Management bei Kontrastmittelzwischenfällen vorträgt. Neben einer möglichen korruptionsrechtlichen Relevanz, legt in derartigen Fällen bereits die MusterBerufsordnung in $\S 32$ Abs. 3 fest, dass die Annahme von Beiträgen Dritter zur Durchführung von Veranstaltungen ausschließlich für die Finanzierung des wissenschaftlichen Programms ärztlicher Fortbildungsveranstaltungen und nur in angemessenem Umfang erlaubt ist. Zudem sind das Sponsoring, dessen Bedingungen und Umfang bei der Ankündigung und Durchführung der Veranstaltung offen zu legen.

Die Durchführung von Fortbildungsveranstaltungen ist für Medizinprodukte- und Pharmahersteller ein geeigneter Rahmen, um für ihre Produkte und Dienstleistungen zu werben. Daher wird die Annahme von Sponsorengeldern durch den veranstaltenden Arzt oder das veranstaltende Krankenhaus analog §20 Abs. 5 des FSA-Kodex nur in einem angemessenen Rahmen gestattet. Damit geht einher, dass der Veranstalter einen Eigenanteil zu tragen oder von den Teilnehmern zu erheben hat. Die Bestimmung eines angemessenen Rahmens ist allerdings schwierig, weil sich die Gegenleistung des Sponsorings, nämlich der damit verbundene öffentlichkeitswirksame Vorteil, praktisch nicht messen lässt. Die Vorschrift will verhindern, dass der Sponsor auf die Ausgestaltung von Veranstaltungen und damit mittelbar auf die Therapieentscheidungen der ärztlichen Zuhörer Einfluss nimmt. Die Anerkennung einer Fortbildungsmaßnahme seitens der Ärztekammern setzt voraus, dass die zu vermittelnden Fortbildungsinhalte frei von wirtschaftlichen Interessen sind und der Veranstalter und die Referenten der Ärztekammer ihre ökonomischen Verbindungen 
zur Industrie offenlegen. Demnach darf sich das Sponsoring lediglich auf die Kosten der eigentlichen Fortbildungsveranstaltung in Form der Raummiete oder der Honorare und Reisekosten für Referenten und nicht auch auf ein etwaiges Rahmenprogramm für Teilnehmer und Begleitpersonen erstrecken, wobei der Veranstalter einen Eigenanteil zu tragen hat. Neben der möglichen berufs- und strafrechtlichen Relevanz eines unangemessen hohen Sponsorings ist ein solches gegebenenfalls auch wettbewerbsrechtlich durch Konkurrenten abmahnbar.

\section{Einladungen zu externen Fortbildungen im Ausland}

Regelmäßig werden Ärzte eingeladen, an internationalen Kongressen teilzunehmen. Neben der Kongressgebühr werden von den einladenden Unternehmen Reise- und Hotelkosten übernommen. Problematisch werden derartige Einladungen dann, wenn neben dem Arzt auch dessen Familie von der Einladung erfasst ist oder die Fortbildung nicht im Vordergrund steht, etwa weil viel Zeit für eigene Aktivitäten zur Verfügung steht. In berufsrechtlicher Hinsicht ist die Teilnahme an dem Jahreskongress unbedenklich, sofern eine darin liegende Annahme eines geldwerten Vorteils lediglich in angemessener Höhe erfolgt. Aus diesem Grund darf die Kostenübernahme nicht über die notwendigen Reisekosten hinausgehen. Nach Auffassung der Berufsordnungsgremien der Bundesärztekammer sind nur das Reisen in der Economy Class und das Wohnen in einem Hotel der gehobenen Mittelklasse notwendig, es sei denn, diese Kategorien sind ausgebucht. Business Class Flüge und die Unterbringung in Luxushotels wären dagegen mangels Erforderlichkeit nicht mehr angemessen. Zusätzlich darf ein Dritter die Tagungsgebühren übernehmen.

Nicht übernommen werden dürfen hingegen Kosten für Familienangehörige, sowie die Kosten für einen über die Veranstaltung hinausgehenden Aufenthalt oder die Kosten für etwaige Freizeitaktivitäten. Insoweit darf es nicht an der wissenschaftlichen Auseinandersetzung der Veranstaltung fehlen, etwa wenn am Tagungsort mehr Zeit für Freizeitaktivitäten als für die Kongressteilnahme zur Verfügung steht. Problema- tisch sind daher Fortbildungsreisen, bei denen der Urlaubs- und Freizeitwert gegenüber dem wissenschaftlichen und beruflichen Wert der Reise in den Vordergrund rückt. Auch wenn eine Fortbildungsveranstaltung im Ausland stattfindet, wodurch mit ihr regelmäßig ein höherer Urlaubsund Freizeitwert verbunden ist, drängt sie jedoch nicht automatisch den wissenschaftlichen und beruflichen Wert der Veranstaltung in den Hintergrund. Erwartungsgemäß findet ein international ausgestalteter Jahreskongress für die Mehrzahl seiner Teilnehmer im Ausland statt. Eine Compliance-Prüfung kann daher nur im konkreten Einzelfall erfolgen.

\section{Staatsanwaltschaften sehen bei Fortbildungssponsoring Anfangsverdacht}

Im Rahmen des Runden Tisches in Thüringen wurde deutlich, dass die Staatsanwaltschaften die Thematik Fortbildung und Einladung zu Fortbildungsveranstaltungen erheblich restriktiver sehen als die Landesärztekammern und einen Anfangsverdacht für ein strafbares Verhalten selbst dann annehmen, wenn die Vorgaben der ärztlichen Berufsordnung eingehalten werden. Die Staatsanwaltschaften sehen danach, entgegen der Regelung in $\S 32$ Abs. 3 der Muster-Berufsordnung den Anfangsverdacht für strafbares Verhalten bereits dann als gegeben an, wenn die Teilnahme an einer Fortbildungsveranstaltung von der Industrie finanziert wird. Ebenso kritisch sieht die Staatsanwaltschaft die Thematik des Veranstaltungssponsorings. Entgegen $\S 32$ Abs. 3 der Muster-Berufsordnung, nach dem ein Sponsoring in angemessenem Umfang erlaubt ist, soll jedwede Annahme von Beiträgen Dritter zur Durchführung von Veranstaltungen den Anfangsverdacht des §299a StGB, unabhängig davon begründen, ob die übernommenen Reisekosten und Tagungsgebühren in einem angemessenen Verhältnis stehen. Sollte sich diese staatsanwaltliche Sichtweise bundesweit durchsetzen, ist Ärzten von der Annahme von Vorteilen durch die Industrie und andere Dritte im Zusammenhang mit Fortbildungen und Einladungen zu Fortbildungsveranstaltungen grundsätzlich abzuraten, da sie andernfalls mit einem
Ermittlungsverfahren rechnen müssen. Dies gilt, wie deutlich geworden ist, auch dann, wenn die berufsrechtlichen Vorgaben eingehalten worden sind.

\section{Fazit}

Das Antikorruptionsrecht hat sich nicht nur in den Strafnormen, sondern auch in den berufs- und sozialrechtlichen Vorschriften niedergeschlagen. Häufig gehen Verstöße gegen Vorschriften des Antikorruptionsrechts Hand in Hand mit abmahnfähigen Wettbewerbsverstößen. Das Bewusstsein für typische Compliance-Risiken muss daher bei der Krankenhausleitung aber auch bei den Ärzten geschärft werden, da möglicherweise bislang übliche und zulässige Verträge und Kooperationen nicht mehr dem aktuellen Antikorruptionsrecht entsprechen. Dies gilt auch im Bereich des Sponsorings im Rahmen von internen und externen Fortbildungsveranstaltungen. Die Schulung von Compliance-Beauftragten auf diesem Gebiet und die Überprüfung bestehender Verträge sind angezeigt.

Ob eine Kooperation tatsächlich gegen Antikorruptions- oder Wettbewerbsrecht verstößt und beendet werden muss oder ob es wohlmöglich nur einer geringfügigen Vertragsanpassung bedarf, ist im Einzelfall zu klären. Auch unterhalb der Strafbarkeitshürde sind Krankenhausleitungen hohen Bußgeldern ausgesetzt, wenn sie möglicherweise inzwischen strafbar gewordene Kooperationen mit anderen Leistungserbringern sowie Pharma- und Medizinprodukteherstellern in Kenntnis der Gesetzesänderungen nicht ernsthaft überprüfen. $\S \S 130,30$ OWiG bestimmen, dass Inhaber eines Betriebes oder Unternehmens, die vorsätzlich oder fahrlässig die Aufsichtsmaßnahmen unterlassen, die erforderlich sind, um in dem Betrieb oder Unternehmen Zuwiderhandlungen gegen Pflichten zu verhindern, die den Inhaber treffen und deren Verletzung mit Strafe oder Geldbuße bedroht ist, ordnungswidrig handeln, wenn eine solche Zuwiderhandlung begangen wird, die durch gehörige Aufsicht verhindert oder wesentlich erschwert worden wäre. Zu den erforderlichen Aufsichtsmaßnahmen gehören auch die Bestellung, sorgfältige Auswahl und Überwachung von Aufsichtspersonen. Die Geldbußen reichen bei 
fahrlässiger Pflichtverletzung bis zu einem Betrag von fünf Millionen Euro, bei vorsätzlichen Pflichtverletzungen sogar bis zu zehn Millionen Euro.

Das Fazit des in Thüringen durchgeführten „Runden Tisches zum Antikorruptionsgesetz“ war, dass die Staatsanwälte die
Tatbestände für den Vorwurf korruptiven Verhaltens im Gesundheitswesen bei Kooperationen zwischen den Beteiligten „sehr weit“ auslegen. Dies sollte alle Beteiligten bei der Überprüfung bestehender und dem Abschluss zukünftiger Vereinbarungen zu großer Achtsamkeit und Zurückhaltung veranlassen.
Prof. Dr. Peter Wigge

Rechtsanwalt

Fachanwalt für Medizinrecht

Ulrike Urbaneck

Rechtsanwältin

Rechtsanwälte Wigge

Scharnhorststraße 40

48151 Münster

$++49 / 251 / 535950$

$++49 / 251 / 5359599$

kanzlei@ra-wigge.de

www.ra-wigge.de 